\title{
Parallel processing of multidimensional stimuli
}

\begin{abstract}
A reaction time (RT) task in which $S$ is required to judge pairs of stimuli either the same or different was used in three experiments in order to determine the processing mode employed in complex discriminations. The results of all experiments indicated that stimulus dimensions are processed by parallel but not necessarily independent analyzers.
\end{abstract}

This report describes a series of experiments designed to investigate the processes involved in discrimination of multidimensional stimuli. More specifically, the studies were designed to provide evidence about the information processing mode of Ss when the information to be processed concerned whether or not two geometric figures were physically the same or different. Egeth (1966) constructed nine possible processing modes that could be used by $S$ s in a same-different $\mathrm{RT}$ task with stimuli varying on one, two, or three binary dimensions. These models were based upon such factors as parallel vs serial processing, random vs fixed order of processing, self-terminating vs exhaustive search, and template matching. His results narrowed the alternatives to two possible modes: serial/self-terminating/random order of search or parallel/self-terminating/ statistically distributed search time. The failure of his results to distinguish between these two alternatives was due to the fact that he assumed $S$ s could ignore irrelevant sources of variation adequately, when, indeed, the data indicated that RTs to relevant dimensions were influenced by variability in irrelevant dimensions.

Nickerson (1966) did a similar experiment using a classification task rather than a same-different matching task. His results concerning parallel and serial processing were also equivocal due to the possible influence of irrelevant information on Ss' focusing on relevant information. Hawkins (1969) reported a series of three experiments using a task similar to Egeth's with two main differences. First, Hawkins varied stimulus complexity across levels of dimensionality by altering the number of physical attributes contained in stimuli, rather than instructing $S s$ to ignore one or more dimensions under some conditions. This change was to eliminate the problem of interference found in Egeth's study. Second, the dependent measure (RT) in
Hawkins's study was based upon a Donder's Type C reaction, in which only one kind of response (either "same" or "different") was made on a block of trials, rather than on a Type B reaction, in which both responses are made within a block of trials. Hawkins reasoned that a Type B reaction may cause response bias and result in the finding of Nickerson (1967) that "same" judgments for three trinary dimensions were faster than the "different" judgments for difference along one dimension. If a search is exhaustive, then Ss should test all three dimensions before making the "same" judgment, and the "same" RT should be at least as slow as the slowest "different" RT.

Hawkins concluded that his findings were totally consistent with none of Egeth's (1966) models, but that the findings are consistent with a model incorporating the following assumptions: (1) Stimulus dimensions are interrogated simultaneously (parallel processing), (2) decision time for any dimension is distributed from trial to trial as a random variable, (3) self-terminating comparisons, and (4) the time required to determine the state of a given dimension covaries with the average time required to determine the states of other dimensions present within a stimulus deck (correlated criteria).

Although Hawkins interprets his results as supporting these conclusions, the results concerning parallel processing and correlated criteria are somewhat equivocal. Assumption 1 concerning parallel processing was supported by data from "same" responses only. That is, Ss gave no "different" responses, and the support for parallel processing was based upon the finding that RTs for judging both of two dimensions "same" was not slower than RT for judging the slowest single dimension "same." Although Ss may interrogate dimensions in parallel when comparing identical stimuli, it is quite possible that a serial search mode may be employed when Ss are required to respond to a difference in any dimension. That is, Ss may divide their attention across all dimensions simultaneously, when they are required to respond, only when stimuli are the same for all dimensions, whereas the requirement of responding to a difference on any dimension may produce focused attention in some serial order. The data presented by Hawkins does not eliminate this possibility. Indeed, his "different" RTs supported a serial model.

Assumption 4 concerning correlated criteria for "same" and "different" judgments was supported by data in which Ss were required to respond "different," and the proportion of differences for each of three dimensions was varied. Hawkins's conclusion was that, as the average discriminability of dimensions contained in a stimulus deck decreases, $S$ s allowed increasingly more time for judgments on all dimensions (correlated criteria). The - suggested interpretation is that a serial model does not fit this data, yet it is quite possible that when the proportion of differences on one dimension is higher than for other dimensions, the $S$ may focus his attention on that dimension first. As the discriminability of the most frequent dimension decreases, the time to make judgments on that dimension first and then go on to other dimensions would increase. Therefore, it would seem that a serial process where the interrogation order is influenced by the most frequently different dimension can also explain the correlated $\overline{\mathbf{R T}}$ result.

The following series of experiments was designed to distinguish between parallel and serial processing modes when both "same" and "different" responses were required of Ss within a block of trials and when interference from irrelevant dimensions was eliminated.

\section{EXPERIMENT 1}

This experiment was designed to determine the relation between the RTs for judging each of three dimensions "different" and the RT for judging all three dimensions "same." Whenever the stimuli on a trial were different, they were different on only one dimension.

\section{Method}

Subjects. Ss in this study consisted of 24 (female) students enrolled in the introductory psychology courses at Kent State University.

Apparatus. A Kodak Carousel 800 projector with an independent light control manufactured by Lehigh Valley Electronics was used to project slides from the rear onto a frosted glass screen. Response apparatus (located in an adjacent room) consisted of a panel containing two response buttons and two feedback lights 
and was situated directly in front of the projection screen at a distance of approximately $5 \mathrm{ft}$. RTs were measured in milliseconds by a Hewlett Packard electronic counter. Recording of RTs, feedback, etc., was accomplished by means of a Lehigh Valley Carousel control panel and Hewlett Packard digital recorder.

Stimulus slide types consisted of pairs of triangles containing three binary stimulus dimensions-color (red or green), dot position (above or below an imaginary midline), and number of inner borders (one or two). The triarigles were $4 \mathrm{in}$. high and $43 / 8$ in. at the base when projected on the screen. The to:a distance covered by the two triangles was 12 in., which corresponded to $11.46 \mathrm{deg}$ of visual angle. The dots were 11/16 in. in diam and the borders were one or two lines $1 / 8$ in. inside the perimeter of the triangle, and $1 / 8$ in. apart when there nere two borders. Stimuli employed were either exactly the same on all dimensions or different on only one of the dimensions, thus eliminating the possibility of interference from differences on other dimensions.

Procedure. $\mathrm{S}$ was seated before the response panel and was instructed verbally concerning the task. A slide that differed on all three dimensions was projected onto the screen during the instructions in order to familiarize $S$ with the dimensions. $S$ was told that pairs of triangles would be shown to her, and that on each pair she was to respond either "same" or "different" by means of the response buttons (response assignment was counterbalanced across Ss to control for hand preference). Throughout the instructions, E emphasized the necessity of responding "as quickly and accurately as possible."

A sequence of 80 same-different slides (20 different on each dimension and 20 same on all dimensions) arranged randomly had been prepared, and was employed for all Ss. Time intervals used were 2-sec stimulus presentation and 2-sec blank period, which served as a ready signal. Following a short practice sequence, measurement trials were begun.

\section{Results and Discussion}

The $\overline{\mathrm{RT}}$ s (mean RTs) for the four stimulus types of same (S), different on dot (dD), different on color (dC), and different on borders (dB) were analyzed by means of a single-factor repeated-measures analysis of variance $(F=49.839, \mathrm{df}=3 / 39$, $\mathrm{p}<.001)$. In addition, Newman-Keuls tests were performed on all pairs of means, indicating that each of the differences between means was significant at the .05 level or beyond. The overall curve relating RT to stimulus type is shown in Fig. 1 .

These results indicate that the discriminability of the three dimensions, as measured by RT to notice a difference on a dimension, was significantly different. The order of RTs was $\mathrm{dC}<\mathrm{dD}<\mathrm{dB}<\mathrm{S}$. The difference in $\overline{\mathrm{RT}}$ s as a function of dimensions indicates a self-terminating search for difference judgments. Same judgments were slower than the slowest difference judgment (dB), thus indicating that "same" was determined after exhausting all potential differences. Since the proportion of different trials compared with same trials was $75: 25$, it is possible that there was a response bias favoring difference judgments, thus accounting for the slow "same" $\overline{R T} .2$ The judgment errors were too few for meaningful comparisons (overall rate was .045). The data of individual $\mathrm{Ss}$ revealed the same ordering of dimensions on the RT variable as the group data. Only four Ss showed reversals in this ordering and these reversals were mainly between $\mathrm{dB}$ and $\mathrm{S}$. Variance attributed to Ss (Ss within trial type) was only .22.

The results of the experiment do not distinguish between a parallel/selfterminating model and a serial/self-terminating model. The obtained order of RTs could reflect the order of interrogation in a serial process or differences in analysis of all dimensions (parallel). The parallel mode implies attention divided between dimensions, whereas the serial mode implies attention focused on individual dimensions in some order. Experiment 2 was designed to distinguish between these two alternatives.

\section{EXPERIMENT 2}

In the first experiment there were three relevant dimensions in the sense that any one of the three could be different on a

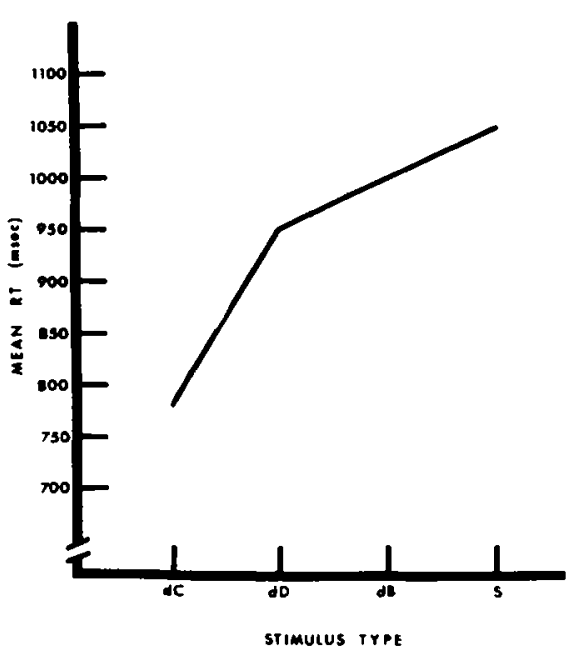

Fig. 1. Mean reaction times for difference judgments on each dimension and for same judgments (Experiment 1). single trial. The reduction of the number of relevant dimensions from three to two should provide further information about processing mode. The parallel and serial models would predict different effects of removing individual dimensions from consideration as potential sources of stimulus difference. If processing is serial, removing a given dimension should have the effect of lowering RTs to dimensions that were interrogated after the given dimension when it was relevant. However, the removal of a dimension interrogated late in the serial order should have no effect on earlier interrogations. For example, the removal of attention to the dot position should lower the "different" $\overline{R T}$ to borders and lower "same" $\overline{\mathrm{RT}}$, whereas it should have no effect on different $\overline{\mathrm{RT}}$ to color. On the other hand, if processing is parallel and the detection of a difference on each dimension is independent of processing on other dimensions, then the removal of a dimension should not affect the "different" $\overline{\mathrm{RT}}$ for the remaining dimensions. However, the removal of the slowest dimension should reduce the "same" $\overline{\mathrm{RT}}$ if "same" comes as a result of the failure to find a difference on the two relevant dimensions. That is, if the "same" judgment always comes after interrogating the slowest dimension, then removal of faster dimensions should not reduce the "same" $\overline{\mathrm{RT}}$. Only removal of the slowest dimension should lower same RTs because the $\mathbf{S}$ no longer has to consider this dimension as a potential source of difference and can make the "same" judgments faster. Experiments 2 and 3 test these predictions.

The three dimensions used in Experiment 1 were used in this study and each dimension was held constant for a block of trials in order to determine its effect on the $\overline{\mathrm{RT}}$ s of the other two dimensions.

\section{Method}

Subjects. The Ss in this study consisted of 12 students (five male, seven female) enrolled in introductory psychology courses.

A paratus. The apparatus was the same as in Experiment 1.

Procedure. Each $\mathbf{S}$ received four blocks of trials. One block of trials was the same as in Experiment 1, with all three dimensions relevant. That is, only one dimension was different when there was a difference but any dimension could be the different dimension. Therefore, there were four stimulus types in this block of trials $(\mathrm{dC}, \mathrm{dD}, \mathrm{dB}$, and $\mathrm{S})$. The other three blocks of trials had only two relevant dimensions each; the third dimension was 
held constant by representing it with a single value on all trials. Each of the three dimensions was held constant for a block of trials and Ss were told before each block which dimensions would be relevant. Within each block, there was an equal number (16) of each of the three trial types. A rest period of $2 \mathrm{~min}$ between each block of trials was given. The sequence of presenting blocks of trials was randomized across Ss.

\section{Results and Discussion}

The $\overline{\mathrm{RT}}$ s for each stimulus type from the three blocks in which one dimension was held constant were compared with the corresponding trial type $\overline{\mathrm{RT}} \mathrm{s}$ from the block where no dimensions were constant. The results are presented in Fig. 2 and analyzed by means of three 2 by 3 analyses of variance with repeated measures on factors. Each analysis of variance contained two conditions (one dimension constant and no dimensions constant) and three stimulus types in each condition. The three stimulus types represent two different judgments (for the two dimensions not held constant) and same judgments.

The analyses revealed that for both the color constant vs nothing constant and the dot constant vs nothing constant analyses, only the main effect of stimulus type was significant $(p<.001)$. There were no differences between the dimension
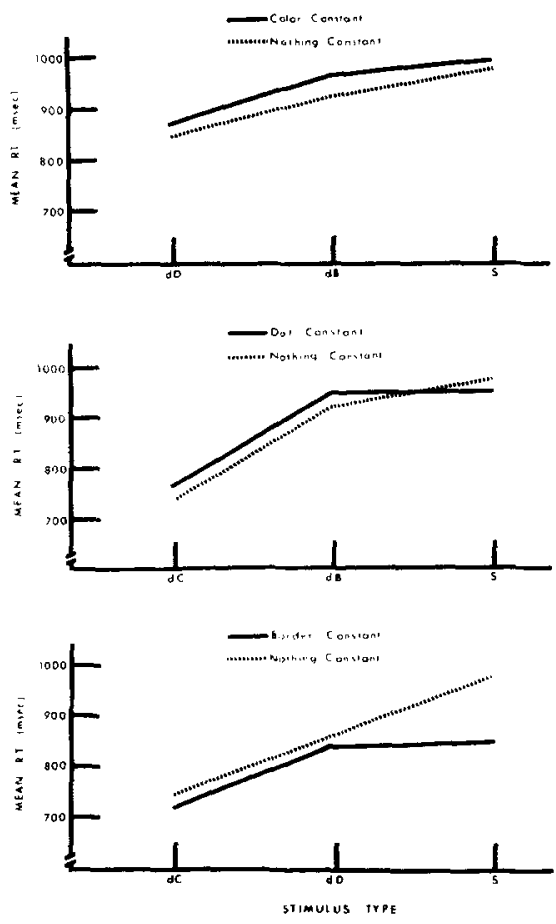

Fig. 2. Mean same and different reaction times for one dimension constant and no dimensions constant (Experiment 2). constant vs no dimension constant conditions for these two analyses. For the border constant vs nothing constant analyses, both the main effect of stimulus type and the Condition (one dimension constant vs no dimensions constant) by Stimulus Type interaction were significant $(p<.001)$. However, Newman-Keuls tests at the various levels of the interaction component showed that only the difference between conditions at same was significant $(p<.01)$. That is, there were no differences between the border constant and nothing constant speeds to discriminate difference along either the color dimension or the dot dimension.

The results of this experiment are completely within the predictions made on the basis of a parallel model. There were no changes in "different" RTs as a function of the dimension held constant and "same" $\overline{\mathrm{RT}}$ changed only when border (the least discriminable dimension) was held constant. The "same" $\overline{\mathrm{RT}}$ s again indicate a self-terminating process and also indicate that Ss could successfully ignore border when it was constant. The "diffe rent" $\overline{R T}$ s also suggested a self-terminating process since the judgment speed was a function of the dimension constituting the difference.

\section{EXPERIMENT 3}

Experiment 2 employed a within-S design in which each $\mathbf{S}$ made judgments on all dimensions. It is possible that the failure to find changes in the "different" $\overline{\mathrm{RT}} \mathrm{s}$ as a function of the dimension held constant was due to Ss' attending to the constant dimension since it may have been relevant previously. That is, Ss may not completely ignore irrelevant dimensions but may make an implicit judgment of same on these dimensions. Although the reduced "same" $\overline{\mathbf{R T}}$ with the slowest dimension constant suggests that Ss are able to ignore this dimension, there is no direct evidence in Experiment 2 that other dimensions are also ignored when irrelevant. A serial process with only partial gating of irrelevant stimuli could explain the results of Experiment 2. In order to test this possibility, the four conditions of Experiment 2 were repeated with a between-S design.

\section{Method}

Subjects. Ss on this study consisted of 24 students (10 males, 14 females) enrolled in the introductory psychology classes.

Apparatus. The apparatus used was the same as that in Experiments 1 and 2.

Procedure. The procedure used was the same as that used in Experiment 2 except that Ss were randomly divided into four groups $(N=6)$ and each group was run under only one condition-color constant, dot constant, border constant, or nothing constant. During instructions, only the dimensions that were relevant for each $S$ were mentioned; the dimensions held constant were never mentioned. Within each block there was an equal number (16) of each of the three trial types.

\section{Results}

The "same" and "different" $\overline{\mathbf{R T}}$ are presented for each condition in Fig. 3. RTs obtained were analyzed by means of three two-factor analyses of variance with repeated measures on one factor. Each analysis compared one of the three groups having one dimension constant to the control group with nothing held constant.

The analysis comparing the color-constant and the nothing-constant groups produced no significant main effect of stimulus type or a significant interaction. The analysis comparing the dot-constant and nothing-constant groups produced a significant istain effect of stimulus type $(F=62.99, \quad d f=2 / 20$, $\mathrm{p}<.001$ ) only. Finally, the analysis comparing border-constant and nothing-constant groups produced a significant main effect of these groups $(F=8.98, \mathrm{df}=1 / 10, \mathrm{p}<.05)$. There was also a significant effect of stimulus type $(F=20.38, d f=2 / 20, p<.001)$. Although the interaction effect was not significant,
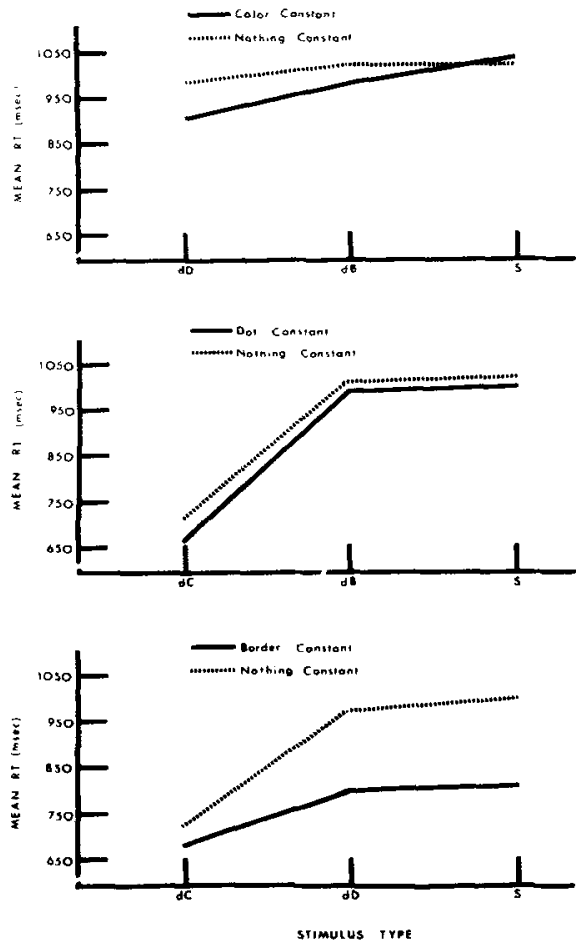

Fig. 3. Mean same and different reaction times for one dimension constant and no dimensions constant (Experiment 3). 
Newman-Keuls tests performed at the three stimulus type levels showed the difference between groups at same to be significant $(p<.01)$ as well as the difference between groups at $\mathrm{dD}(\mathrm{p}<.05)$.

These results are quite in agreement with the results of Experiment 2 in that, even in a between-Ss design, the removal of a given dimension does not significantly change the RTs to the remaining dimensions when compared to a group with all three dimensions relevant. The only inconsistency appcars to be in the border-constant group in which a significant effect of group was found, athough the a posteriori tests indicate that, again, much of the effect can be accounted for by the difference between groups at same. Again, the parallel processing model would predict this decrease in same RT for the border-constant group. Even though the dD RTs were different between border-relevant and border-constant conditions, a serial nodel would not predict this change. The serial model would predict a reduction in border $\overline{\mathrm{RT}}$ with dot constant. One possible explanation of the reduction of the $\mathrm{dD} \overline{\mathrm{RT}}$ with border constant is consistent with the correlated
RT assumption of Hawkins. That is, the presence of the border as a relevant dimension may increase the RT cirterion for dot and the absence of border reduces the judgment criterion for dot. However, since this effect was not manifest in the data of Experiment 2, this interpretation is, at best, tenuous.

\section{DISCUSSION}

The results of the three studies taken together strongly support a parallel processing mode. None of the data are consistent with a serial model and none of the results contradict the four assumptions of Hawkins presented in the introduction to this report. Same judgments came after the slowest relevant dimension in all cases. This finding may be due to the $75: 25$ proportion of different to same stimuli, but the interpretation of the processing mode gained from "different" $\overline{\mathrm{RT}}$ s is not affected by proportion differences (see Note 2). At any rate, these studies and the Hawkins studies indicate that individuals can process multidimensional information with paralle1 (but not necessarily independent) channels.

\section{REFERENCES}

EGETH, H. E. Parallel versus serial processes in multidimensional stimulus discrimination. Perception \& Psychophysics, 1966, 1, 245-252.

HAWKINS, H. L. Parallel processing in complex visual discrimination. Perception \& Psychophysics, 1969, 5, 56-64.

NICKERSON, R. S. Response times with a memory-dependent decision task. Journal of Experimental Psychology, 1966, 72, 761-769.

NICKERSON, R. S. "Same-different" response times with multi-attribute stimulus differences. Perception \& Motor Skills, 1967, 24, 543-554.

\section{NOTES}

1. Address: Kent State University, Kent, Ohio 44240.

2. In a pilot study it was determined that a 75:25 proportion of different and same trials compared to a 50:50 proportion of different and same trials produced very similar "different" RTs. However, the "same" RTs for the 50:50 proportion were significantly lower than with the $75: 25$ proportion. Since the main in terest here is in the difference judgnients and they are unaffected by these proportions, a 75:25 proportion was used throughout the three experiments.

(Accepted for publication October 27, 1969.) 